\title{
Needle Geometry, Target Migration and Substrate Interactions in High Resolution
}

\author{
Matthew Oldfield, Alexander Leibinger, Pia-Afra Kaufmann, \\ Marine Bertucchi, Frank Beyrau and Ferdinando Rodriguez y Baena ${ }^{1}$
}

\begin{abstract}
Recent investigations considering flexible, steerable needles for minimally invasive surgery have shown the significance of needle shape in determining the needle-tissue interactions leading to the access of targets. Digital Image Correlation has enabled internal deformation and strain caused by needle insertions to be seen in a soft tissue phantom at high resolution for the first time. Here, the impact of tip design on strains and displacements of material around the insertion axis is presented using Digital Image Correlation in a stable, plane-strain configuration. Insight into the shape of needles to minimise tissue trauma and generate interactions that would enable optimal steering conditions is provided. Needle tips with an included bevel angle up to $40^{\circ}$ result in asymmetric displacement of the surrounding tissue phantom. Increasing the included tip angle to $60^{\circ}$ results in more predictable displacement and strains that may enhance steering forces with little negative impact on the phantom.
\end{abstract}

\section{INTRODUCTION}

In recent years significant interest has been shown in the mechanics of needle insertions [1]. Needle-tissue interaction mechanics are critical in the development of steerable needles able to navigate curved trajectories with the aim of targetted therapy delivery or diagnosis. Some steerable needles can work in a cavity [2], [3], but many rely on the interactions between a relatively flexible shaft, tip and tissue substrate [4], [5]. The impact on trajectory of the needle geometry, particularly that of the needle tip, interacting with the substrate has been investigated through parametric experiments [6]. However, an in-depth understanding of the interaction mechanics has not been provided. Different steerable needles, coupled with their actuation strategies, can also result in varying degrees of tissue damage [7] - a side effect that needs to be understood.

A multi-part needle, using axially-interlocked segments, is capable of steering along trajectories of multiple curvatures [8], [9]. Like other steerable needles, the curved trajectory (Fig. 1) results from a flexible material and bevelled tip geometry for each of the individual needle segments. Previous experiments in a seeded, gelatin, soft tissue phantom, using equipment and methods associated with particle image velocimetry (PIV), have revealed the needle-substrate interaction mechanics in unprecedented detail [10]. It has been shown that different actuation mechanisms of the four

The research leading to these results has received funding from the European Research Council under the European Union's Seventh Framework Programme (FP7/2007-2013) / ERC grant agreement $\mathrm{n}^{\circ}$ 258642-STING.

${ }^{1}$ All authors are with the Department of Mechanical Engineering, Imperial College London, South Kensington, London, SW7 2AZ, UK. f.rodriguez@imperial.ac.uk

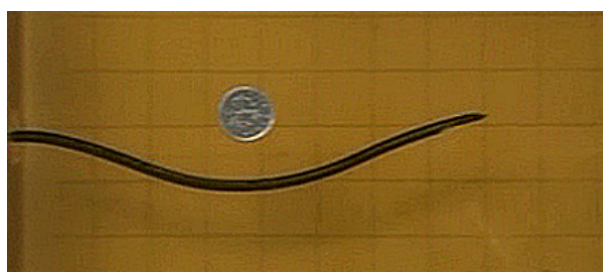

Fig. 1. A $4 \mathrm{~mm}$ diameter, bevel-tipped flexible needle based on [8], steering with three bends in gelatin around an $18 \mathrm{~mm}$ diameter obstacle. Minimum radius of curvature is approximately $70 \mathrm{~mm}$.

needle segments may reduce substrate strain and, hence, tissue trauma [11].

The ability to see displacements and strains at the needlesubstrate interface is an invaluable tool. Other experimenters have shown the needle-substrate interface in detail [12] without quantifiable data on the substrate condition. Shergold and Fleck [13] have demonstrated, retrospectively, that the mode of substrate failure depends on the tip geometry. Here, it is assumed that the excessive shearing of mode-II failure is undesirable. It is therefore important to analyse the impact of needle geometry on both tissue trauma and steering.

An investigation of the insertion mechanics of different tip designs in a gelatin soft tissue phantom is presented. A number of shortcomings of previous studies are overcome as follows: the equipment commonly required for PIV is replaced by an economical rig suited to low displacement rates; out of plane motion of the substrate around a cylindrical needle is removed by analysing the geometry under plane strain conditions; a range of needle tip designs are analysed to quantify high-resolution substrate mechanics and to demonstrate effects leading to tissue damage and needle steering capabilities.

A brief outline of theoretical considerations for digital image correlation (DIC) follows. The experimental rig, processing steps and experimental parameters are descibed in Section III. Quantified results for the blade-tissue interactions are presented and discussed in Sections IV and V. Conclusions and future work are provided in Section VI.

\section{THEORY}

The theory behind PIV is well established and covered in [14]. Here, using DIC, the process to go from correlated displacements between images to a strain in the phantom is outlined. A low displacement rate condition is assumed in the tissue phantom. Using the approach described in [11] the $2 \mathrm{D}$ position vector, $\mathbf{x}$, is originated at the fixed 
positions of the DIC measurement grid. These material points are then tracked over the imaged frames by interpolating the displacements on the DIC measurement grid to give a displacement vector, $\mathbf{u}$, at $\mathbf{x}$ :

$$
\left\{\mathbf{x}_{n+1}\right\}=\left\{\mathbf{x}_{n}\right\}+\left\{\mathbf{u}_{n}\right\}
$$

where $n$ defines a particular image in the recorded sequence.

Using a triangular mesh, where the vertices correspond to the tracked material points, the deformation gradient, $\mathbf{F}$, of each element in the mesh can be established by comparing the deformed and undeformed configurations of corresponding element sides [15]. For each element, the Green-Lagrange strains can be established using $\mathbf{F}$ and the identity matrix, I:

$$
\epsilon=\left[\mathbf{F}^{T} \mathbf{F}-\mathbf{I}\right]
$$

As the superimposed elements have the same volume, and the phantom is assumed incompressible, the strain energy per element, $U$, can be calculated using $\epsilon$ and the constitutive relationship between stress and strain, $\mathbf{E}$, where $E$ is the Young's modulus and $\nu$ is Poisson's ratio:

$$
\mathbf{E}=\frac{E}{1-\nu^{2}}\left[\begin{array}{ccc}
1 & \nu & 0 \\
\nu & 1 & 0 \\
0 & 0 & \frac{(1-\nu)}{2}
\end{array}\right]
$$

and

$$
U=\frac{1}{2} \epsilon^{T} \mathbf{E} \epsilon
$$

\section{METHOD}

\section{A. Soft Tissue Phantom Preparation}

Bovine gelatin with a bloom strength of 250 (Sleaford Quality Foods, Sleaford, UK) was added to water, using $3.5 \%$ by mass at $60 \pm 10^{\circ} \mathrm{C}$, to give a transparent phantom with elasticity comparable to humain brain tissue. Aluminium Oxide $\left(\mathrm{Al}_{2} \mathrm{O}_{3}\right)$ powder, $1 \mathrm{~g}$, with a particle size of 5 microns was stirred into the liquid gelatin mixture. The gelatin mixture was poured to a depth of $78 \pm 1 \mathrm{~mm}$ in containers with an $80 \mathrm{~mm} \times 80 \mathrm{~mm}$ cross section before cooling overnight at $11-13^{\circ} \mathrm{C}$. Before blade insertions, the gelatin was left to reach a surface temperature of $\left(20 \pm 1^{\circ} \mathrm{C}\right)$.

\section{B. Experimental Hardware}

In the experiments presented here, blades $130 \mathrm{~mm}$ long, $4 \mathrm{~mm}$ high and $50 \mathrm{~mm}$ deep and were inserted into the gelatin phantom. Deep blades, as opposed to needles, enabled reliable plane-strain assumptions to be made about the imaged deformation profile. The blades were manufactured from ABS-M30 and had symmetric tips with $20^{\circ}, 40^{\circ}, 60^{\circ}, 80^{\circ}$ and $120^{\circ}$ included angles. A motor and encoder (Maxon A$\max )$ were used to control the insertion of the blades into the gelatin block at a rate of $1 \mathrm{~mm} / \mathrm{s}$.

A laser light sheet less than $0.5 \mathrm{~mm}$ deep $(4.5 \mathrm{~mW} 532 \mathrm{~nm}$ collaminated DPSS laser, Thorlabs Ltd., Ely, UK) was generated along the insertion axis using the following sequence of lenses: plano convex; plano concave; plano convex (Fig. 2). The light sheet entered the gelatin phantom at the unconstrained end of the sample and was sufficiently strong to
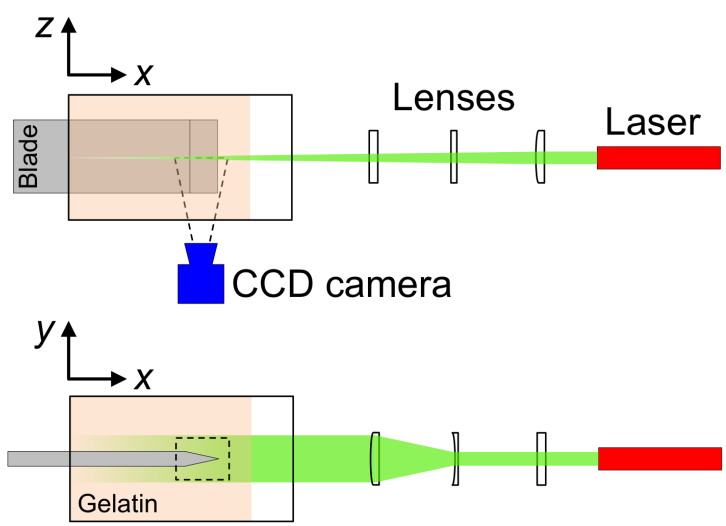

Fig. 2. Experimental schematic showing key equipment and the lens configuration to generate a laser sheet in the seeded phantom

provide a correlatable scatter from $\mathrm{Al}_{2} \mathrm{O}_{3}$ particles over the full camera field of view.

\section{Image Acquisition and Post Processing}

Images of the particles in the laser light sheet were captured using a CCD camera (AVT Guppy Pro F-146B, Stadtrod, Germany) at a rate of $5 \mathrm{fps}$. A field of view $20.2 \mathrm{~mm} \times 15.1 \mathrm{~mm}$ was calibrated using a test grid positioned in set gelatin. Distortion of the image was negligible.

Post-processing of the images was performed using the PIVlab toolbox (www.pivlab.blogspot.co.uk) in Matlab (Mathworks). A double-pass correlation algorithm using windows of 32 then 16 pixels with a $50 \%$ overlap was implemented and conservative smoothing of the vector fields was applied retrospectively. The blade was masked during the correlation process and also during the application of (1). From an image size of $1024 \mathrm{px} \times 768 \mathrm{px}$ displacement vectors were obtained on a $47 \times 63$ grid. Rigid body motion tests of the seeded gelatin at $1 \mathrm{~mm} / \mathrm{s}$ verified a sufficient amount and distribution of $\mathrm{Al}_{2} \mathrm{O}_{3}$ remained in the gelatin after setting to perform satisfactory DIC.

\section{REsUlts}

An example of an image extracted during the blade insertions showing the blade itself and scatter from $\mathrm{Al}_{2} \mathrm{O}_{3}$ particles is given in Fig. 3. Typical displacement vector fields

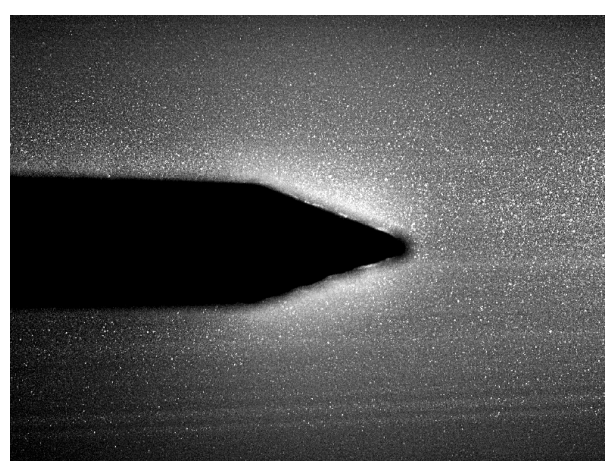

Fig. 3. Typical image for DIC with $\mathrm{Al}_{2} \mathrm{O}_{3}$ particles scattering light around a blade with a tip angle of $40^{\circ}$ 

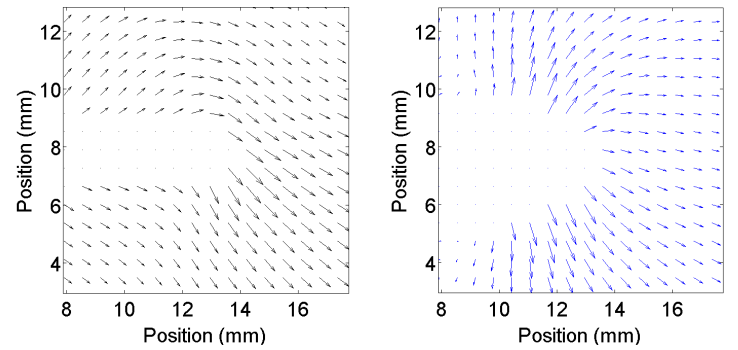

Fig. 4. Displacement vectors for a $20^{\circ}$ blade (left) and $60^{\circ}$ blade showing asymmetric and symmetric material motion respectively at the tip

around $20^{\circ}$ and $60^{\circ}$ blade tip angles are shown in Fig. 4. For the $20^{\circ}$ blade the response is far less symmetric and the phantom 'wraps' around the tip during insertion.

Strain profiles, such as that in Fig. 5 for $\epsilon_{x x}\left(\epsilon_{11}\right.$ in (2)) show 'lobes' of compressive strain around the blade bevels and tension behind the tip. To smooth the response, element strains are averaged at their connecting vertices.

Displacement profiles in Fig. 6, calibrated against the appearance of the blade tip, were used to track the axial migration of a material point $1 / 3$ of the way into the field of view on the blade insertion axis . Six insertions each for blade angles $20^{\circ}, 40^{\circ}, 60^{\circ}$ and $80^{\circ}$ are shown. If the blade outpaced the material movement, subsequent measurements are removed from the analysis. No significant difference exists between geometries, but the $40^{\circ}$ blade tends to cause greater migration when the blade is approaching than its $60^{\circ}$ counterpart.

The same set of blade insertions have been used to generate a comparison of the mean strain energy in the field of view using (4). There appears to be little significant difference between the blade geometries except that strain energies from the $80^{\circ}$ blade tend to higher values than the $60^{\circ}$ blade (Fig. 7).

In all tests there was a large degree of scatter between results from the same blade geometries. This has made obtaining statistical significance of the output impossible and shows the variablility that can result from highly resolved spatial measurements in gelatin.

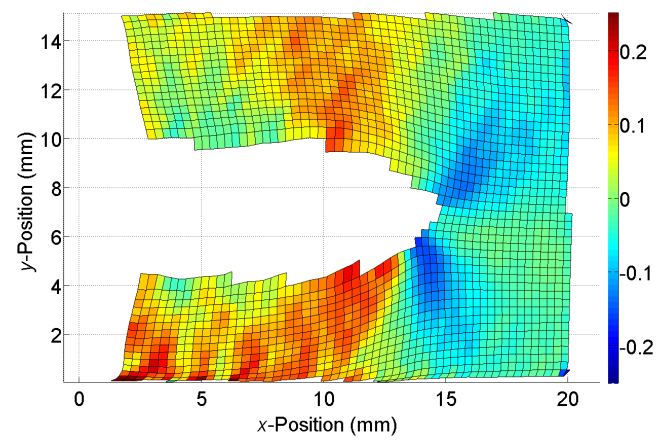

Fig. 5. $\epsilon_{x x}$ field generated around a $60^{\circ}$ blade tip. 'Lobes' of strain indicate a material condition tending towards mode-I failure.

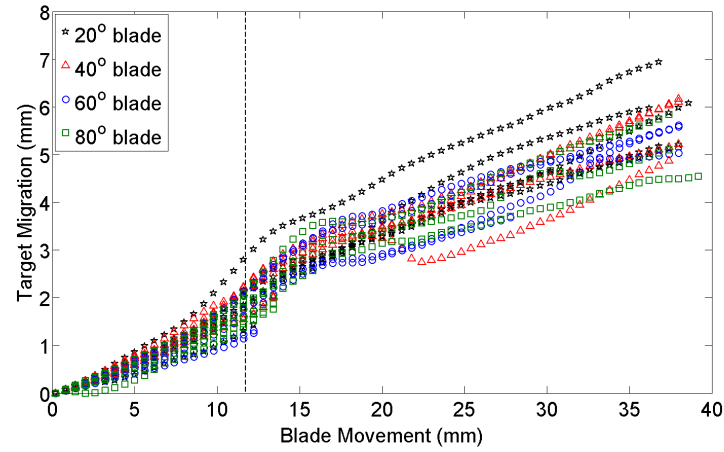

Fig. 6. Axial movement of material points initially on the insertion axis, indicating that target migration is not significantly affected by blade geometry. The dashed line represents the arrival of the blade tip at the measurement point.

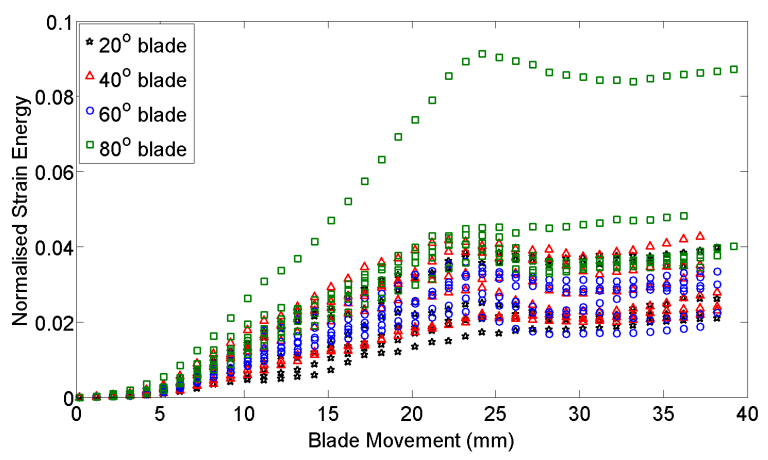

Fig. 7. The mean strain energy, normalised using a nominal $E$, showing the transition to potentially greater tissue damage with an $80^{\circ}$ blade tip

\section{Discussion}

Previous work [10], [11] has shown the applicability of the DIC technique to studying needle insertions in a tissue phantom. Due to the low velocity of these insertions it is proposed that an economical experimental setup is capable of delivering DIC at the same resolution. This is confirmed with DIC succeeding on a plane $40 \mathrm{~mm}$ inside a gelatin phantom, with output vectors obtained every $0.32 \mathrm{~mm}$. The total equipment cost is an order of magnitude lower than that for the type of camera conventionally used to perform PIV, making the method accessible for a wide variety of low displacement rate biomedical investigations.

High resolution output on a plane inside the gelatin phantom shows the technique is transferable to other transparent materials. The homogeneity of gelatin is ideal for design evaluation but, necessarily, lacks the mechanical variability of biological tissue. Surface imaging can be used for material characterisation [16] but internal mechanics are not available at equivalent resolutions in opaque tissues. Gelatin offers the compromise of similar elasticity but greater friction than is likely to be seen in soft tissues with a high liquid content. Observed phantom behaviour will, therefore, be moderated for tissues - as would be the case when testing in all particular substrates.

Non-invasive study of target migration during rigid blade 
insertions has been quantified with unprecedented accuracy. The high resolution also makes this DIC method idealy suited to the quantification of the mechanics of conventional small diameter surgical needles. Complex mechanical interactions crucial to the performance of steerable needles and, for example, the palpation of phantoms with inclusions, could be directly compared with numerical studies. Addition of further light sources and manipulation of the optics has potential for economical measurements in multiple planes or axes.

In the context of steerable needles introduced in Section I, the DIC experimental setup has enabled five different bevel tips to be investigated. For all insertions, the blade with an included angle of $120^{\circ}$ resulted in too much damage of the gelatin phantom to perform DIC effectively. All of the other blades gave good results but with some tests rejected based on local damage of the substrate interfering with DIC.

Figure 7 demonstrates that there is little difference between the strain energy seen in the tissue phantom for most blade angles. A trend towards higher values suggests that the blade with an $80^{\circ}$ angle would be more harmful if equating high strains with greater tissue damage. A benefit of this clustering of strain energy for all other blade types is that greater steering forces associated with a given tip geometry [4], [6] do not seem to come at the cost of increased tissue damage for included angles up to $60^{\circ}$. Figure 4 shows the deformation profile is more symmetrical around blades tips of $60^{\circ}$ rather than, for example, $20^{\circ}$. This is attributed to the difficulty in reaching the conditions required for material failure with more acute blades and unbroken phantom material being dragged around the needle tip. It is proposed here that larger blade angles result in a better cleaving of the gelatin phantom by facilitating mode-I failure. Distinct 'lobes' of strain around tips with larger bevel angles suggest material conditions suited to this type of cutting.

Without the detailed, quantifiable and internal data provided by DIC, this type of interaction can only be speculative. Clear deformation plots, evidence of target migrations and strain fields show that larger included angles that may facilitate steering do not, necessarily, produce greater damage. Here, an upper limit of a $60^{\circ}$ included angle can be assumed and, due to observed damage, an included angle of $120^{\circ}$ results in significant damage and a likely change in cutting mode - though this cannot be quantified due to the resulting difficulty in correlating images. The economical DIC experiment has, therefore, provided a vital insight into needle bevel geometry for future design iterations and validation of numerical models.

\section{CONCLUSIONS AND FUture WORK}

It has been shown that, in a transparent tissue phantom, highly resolved displacement and strain fields can be measured with an economical experimental configuration suited to low speed deformations. Output from DIC experiments has been achieved on a grid with spacing less than $0.35 \mathrm{~mm}$.

Measurements of strain and displacement around blades with different tip geoemetries show target migration and strain energy are insensitive to blade profile. The experiment suggests that blade tip angles $80^{\circ}$ and greater are likely to cause more damage. Blade angles of $20^{\circ}$ and $40^{\circ}$ result in neither greater cutting capability nor reduced target migration, while blade tip angles of $60^{\circ}$ result in a more symmetric phantom response and controlled cutting process.

Future work will explore the use of more complex tip profiles to optimise both cutting and tissue separation. Developing the experiment to give measurements in multiple planes and dimensions will also enhance our understanding of needle insertion mechanics.

\section{REFERENCES}

[1] D. J. van Gerwen, J. Dankelman, and J. J. van den Dobbelsteen, "Needle-tissue interaction forces - a survey of experimental data," Medical Engineering \& Physics, vol. 34, no. 6, pp. 665 - 680, 2012.

[2] R. Webster, J. Romano, and N. Cowan, "Mechanics of precurved-tube continuum robots," Robotics, IEEE Transactions on, vol. 25, no. 1, pp. 67-78, 2009

[3] P. Dupont, J. Lock, B. Itkowitz, and E. Butler, "Design and control of concentric-tube robots," Robotics, IEEE Transactions on, vol. 26, no. 2, pp. 209-225, 2010.

[4] S. Misra, K. Reed, B. Schafer, K. Ramesh, and A. Okamura, "Mechanics of flexible needles robotically steered through soft tissue,' The International Journal of Robotics Research, vol. 29, no. 13, pp 1640-1660, 2010

[5] C. Lehocky, X. Li, and C. Riviere, "Design and control considerations for safe needle steering in brain tissue," in Communications, Computing and Control Applications (CCCA), 2012 2nd International Conference on, 2012, pp. 1-6.

[6] A. Majewicz, T. Wedlick, K. Reed, and A. Okamura, "Evaluation of robotic needle steering in ex vivo tissue," in Robotics and Automation (ICRA), 2010 IEEE International Conference on, 2010, pp. 20682073.

[7] P. Swaney, J. Burgner, H. Gilbert, and R. Webster, "A flexurebased steerable needle: High curvature with reduced tissue damage," Biomedical Engineering, IEEE Transactions on, vol. 60, no. 4, pp. 906-909, 2013.

[8] R. Secoli and F. Rodriguez y Baena, "Closed-loop 3d motion modeling and control of a steerable needle for soft tissue surgery," in Robotics and Automation (ICRA), 2013 IEEE International Conference on, 2013, pp. 5831-5836.

[9] S. Y. Ko, L. Frasson, and F. Rodriguez y Baena, "Closed-loop planar motion control of a steerable probe with a "programmable bevel" inspired by nature," Robotics, IEEE Transactions on, vol. 27, no. 5, pp. 970-983, 2011.

[10] J. Kerl, T. Parittotokkaporn, L. Frasson, M. Oldfield, F. R. y Baena, and F. Beyrau, "Tissue deformation analysis using a laser based digital image correlation technique," Journal of the Mechanical Behavior of Biomedical Materials, vol. 6, pp. 159-165, 2012.

[11] M. Oldfield, C. Burrows, J. Kerl, L. Frasson, T. Parittotokkaporn, F. Beyrau, and F. Rodriguez y Baena, "Highly resolved strain imaging during needle insertion: Results with a novel biologically inspired device," Journal of the Mechanical Behavior of Biomedical Materials, vol. 30, pp. 50-60, Feb. 2014.

[12] Y. R. van Veen, A. Jahya, and S. Misra, "Macroscopic and microscopic observations of needle insertion into gels," Proceedings of the Institution of Mechanical Engineers, Part H: Journal of Engineering in Medicine, vol. 226, no. 6, pp. 441-449, 2012.

[13] O. A. Shergold and N. A. Fleck, "Experimental investigation into the deep penetration of soft solids by sharp and blunt punches, with application to the piercing of skin," Journal of Biomechanical Engineering, vol. 127, no. 5, pp. 838-848, 2005.

[14] M. Raffel, C. E. Willert, and J. Kompenhans, Particle image velocime try: a practical guide. Berlin: Springer Verlag, 1998.

[15] J. Teran, S. Blemker, V. N. T. Hing, and R. Fedkiw, "Finite volume methods for the simulation of skeletal muscle," in Proceedings of the 2003 ACM SIGGRAPH/Eurographics symposium on Computer animation, ser. SCA '03. Aire-la-Ville, Switzerland, Switzerland: Eurographics Association, 2003, pp. 68-74.

[16] H. Kwon, A. D. Rogalsky, and D.-W. Kim, "On the measurement of fracture toughness of soft biogel," Polym Eng Sci, vol. 51, no. 6, pp. 1078-1086, 2011. 